\title{
A pessoa como centro do cuidado na prática do médico de família
}

\author{
The person at the center of care in the practice of the family doctor
}

\section{La persona en el centro de la atención en la práctica del médico de familia}

José Mauro Ceratti Lopes. Serviço de Saúde Comunitária do Grupo Hospitalar Conceição (SSC-GHC). Universidade Federal de Ciências da Saúde (UFCSPA). Porto Alegre, RS, Brasil. jmauro.lopes@terra.com.br (Autor correspondente)

Jorge Alberto Rosa Ribeiro. Faculdade de Educação da Universidade Federal do Rio Grande do Sul (FACED/UFRGS). Porto Alegre, RS, Brasil. jorge.ribeiro@ufrgs.br

\section{Resumo}

Objetivo: uma atuação centrada na pessoa é considerada essencial para o médico de família e comunidade considerando-se as mudanças ocorridas na sociedade nos séculos XIX e XX. Este artigo apresenta um estudo de caso, cujo objetivo foi identificar se os componentes que caracterizam inequivocamente 0 Método de Abordagem Clínica Centrada na Pessoa (MCCP) estão presentes na prática do médico de família. Métodos: os dados foram coletados por meio de entrevistas com médicos de família e com pessoas por eles atendidas, sendo os depoimentos gravados, transcritos e analisados com base no Discurso do Sujeito Coletivo. Resultados: identificaram-se aspectos comuns ou contraditórios nos discursos de pessoas e médicos sobre a aplicação da Abordagem Centrada na Pessoa. Conclusão: ainda não existe por parte dos médicos de família um conhecimento adequado sobre significado e aplicação de uma Abordagem Clínica Centrada na Pessoa.

\section{Abstract}

Objective: a person-centered practice is essential to the family and community doctor in the face of changes in society in the nineteenth and twentieth centuries. This article reports on a case study whose objective was to identify if the components that uniquely characterize the Person Centered Clinical Approach are present in the practice of the family doctor. Methods: data were collected through interviews with family doctors and with people seen by them. The interviews were recorded, transcribed and analyzed using the Discourse of the Collective Subject. Results: the study identified common or contradictory discourses of people and doctors on the application of the person-centered approach. Conclusion: family doctors still lack adequate knowledge about the meaning and application of the Person-Centered Clinical Approach.

\section{Resumen}

Objetivo: una actividad centrada en la persona es esencial para el médico de familia y comunidad frente a los cambios en la sociedad en los siglos XIX y XX. Este artículo es un estudio de caso, cuyo objetivo fue identificar si los componentes que caracterizan de forma exclusiva el método de la Clínica de Enfoque Centrado en la Persona (MCCP) están presentes en la práctica del médico de familia. Métodos: los datos fueron recolectados a través de entrevistas con los médicos de familia y las personas atendidas por ellos. Las entrevistas fueron grabadas, transcritas y analizadas utilizando el Discurso del Sujeto Colectivo. Resultados: el estudio identificó los discursos comunes o contradictorios de las personas y de los médicos sobre la aplicación del enfoque de los aspectos centrados en la persona. Conclusión: no existe por parte de los médicos de familia adecuado conocimiento del significado y de la aplicación del Enfoque Centrado en la Persona.
Palavras-chave:

Medicina de Família e

Comunidade

Assistência Centrada no Paciente Relações Médico-Paciente

\section{Keywords:}

Family Practice

Patient-Centered Care

Physician-Patient Relations

\section{Palabras clave:}

Medicina Familiar y Comunitaria Atención Dirigida al Paciente Relaciones Médico-Paciente 


\section{Introdução}

The good physician treats the disease but the great physician treats the patient who has the disease. Sir William Osler

A principal tarefa da medicina no século XXI será a descoberta da pessoa. Ela terá que encontrar as origens da doença e do sofrimento e, com esse conhecimento, desenvolver métodos para aliviar a dor, diminuir danos e reduzir o sofrimento. Ao mesmo tempo, deverá revelar o poder da própria pessoa, assim como nos séculos XIX e XX foi revelado o poder do corpo. ${ }^{1}$ Uma atuação centrada na pessoa é considerada fundamental para um bom desempenho de qualquer profissional da área da saúde, mas ao médico de família e comunidade ela é imprescindível, considerando-se as mudanças ocorridas na sociedade nos séculos XIX e XX. Embora algumas dessas mudanças tenham facilitado a relação do médico com as pessoas, outras a dificultaram. ${ }^{2}$ Soma-se a isso a falência dos modelos convencionais de assistência e educação médica, que deveriam dar conta das necessidades da prática diária, mas que, de fato, são incompletos e pouco abrangentes. Com isso, torna-se necessário utilizar uma abordagem para os problemas de saúde que seja capaz de reduzir a insatisfação das pessoas e a frustração dos médicos, assim como de proporcionar um cuidado adequado.

Segundo Stewart, ${ }^{2}$ existem trabalhos mostrando que uma atuação centrada na pessoa apresenta resultados positivos quando comparada aos modelos tradicionais, já que aumenta a satisfação da pessoa, melhora a aderência aos tratamentos, reduz preocupaçóes, reduz sintomas e melhora a situação fisiológica. Também estudos realizados por Little ${ }^{3}$ concluem que na atenção primária as pessoas desejam fortemente uma abordagem centrada na pessoa, com comunicação, parceria e promoção da saúde. Além disso, os médicos devem ser sensíveis e atentos a quem tem preferência por este modelo de abordagem pessoas mais vulneráveis psicossocialmente ou que estão se sentindo particularmente mal. $\mathrm{O}$ autor conclui também que a percepção por parte de quem é atendido acerca dos componentes da abordagem centrada na pessoa pode ser medida com segurança e predizer diferentes resultados, e que se os médicos não proporcionarem uma abordagem centrada na pessoa, esta vai sentir-se menos satisfeita, menos capaz, podendo ter sintomas agravados e com altas taxas de encaminhamento.

Além desses fatos, a prática do médico de família e comunidade, para alcançar uma atuação de acordo com os princípios ${ }^{4,5}$ que regem esta especialidade, deve utilizar o método de atendimento clínico centrado no paciente (MCCP) como modelo para abordagem integral aos problemas de saúde. ${ }^{2}$

Embora a prática médica no Brasil já apresente uma postura mais humanizada e integral ao tratar dos problemas das pessoas, de modo geral ainda não conseguiu romper com o método de abordagem aos problemas de saúde baseado no modelo biomédico tradicional, que tem como características principais ser centrado na doença e no médico. Mesmo com todo o progresso trazido pelo desenvolvimento tecnológico e do conhecimento, o evento central da vida profissional do médico, e especialmente do médico de família, continua sendo a consulta e, por consequência,ela torna-se o ato principal do seu processo de trabalho. Surge entáo o desafio para a medicina contemporânea de integrar dentro do processo de produçáo de cuidado a medicina baseada em evidências, o atendimento centrado na pessoa e o trabalho em equipe.

Para dar início a este processo, e considerando o ponto de vista das pessoas (qual é a percepção do cuidado que recebe?) e dos médicos (o quanto conhece e percebe sua atuaçáo centrada na pessoa?), realizamos pesquisa com o objetivo de identificar se médicos de família integrantes de um serviço de referência como modelo assistencial e centro formador dominam o conhecimento e a aplicaçáo dos componentes essenciais do método clínico centrado na pessoa, analisados do ponto de vista de quem presta o atendimento e de quem foi atendido.

\section{Metodologia}

Foi desenvolvida pesquisa qualitativa, através de um estudo de caso, ${ }^{6}$ utilizando-se entrevistas semiestruturadas para coleta das informaçóes. As entrevistas foram gravadas com gravador de voz, transcritas e analisadas. O caso estudado é o relacionamento dos médicos de família e comunidade com as pessoas por eles atendidas em três unidades de saúde no Grupo Hospitalar Conceição (SSC-GHC), em 2004/2005. Foi realizada revisão de literatura, nacional e internacional, visando fundamentar teórica e analiticamente o estudo. O modelo de análise utilizou como instrumento o "Discurso do Sujeito Coletivo"(DSC), que é um discurso-síntese elaborado com pedaços de discursos de sentidos semelhantes reunidos num só discurso. Tendo como fundamento a teoria da Representação Social e seus pressupostos sociológicos, o DSC é uma técnica de tabulação e organização de dados qualitativos que resolve um dos grandes impasses da pesquisa qualitativa, na medida em que permite, por meio de procedimentos sistemáticos e padronizados, agregar depoimentos sem reduzi-los a quantidades. ${ }^{7}$ 
Os entrevistados pertenciam a duas categorias: médicos de família e comunidade e pessoas por eles atendidas. Foram entrevistados cinco médicos de três Unidades de Saúde e 14 pessoas por eles atendidas. Com este número, obtivemos saturação necessária para o estudo. A "saturação necessária" foi obtida no momento em que as respostas dos entrevistados sobre as diversas questôes abordadas tornaram-se repetitivas, não acrescentando fatos novos. A escolha por estas três Unidades deveu-se ao fato de as mesmas contarem com profissionais que estão desde os primeiros tempos de implantação do SSC-GHC e serem os locais onde ocorreu menor número de transferências ou substituiçóes de profissionais médicos.

Escolheu-se por conveniência um dia da semana para a visita à Unidade, momento quando se perguntou aos médicos presentes se estes estavam dispostos a participar da pesquisa. Se havia mais de um médico interessado, era realizado sorteio. As pessoas atendidas por estes médicos também eram convidadas a participar. Todos os entrevistados, tanto médicos como as pessoas atendidas, assinaram termo de consentimento.Para se alcançar a saturação e, assim, elaborar o DSC, foi estabelecido previamente que o número de pessoas entrevistadas seria de duas a três pessoas da agenda de cada médico, seguindo a ordem de atendimento e o aceite em participar. Caso fosse necessário, mais pessoas seriam entrevistadas.

As entrevistas seguiram roteiros semiestruturados específicos para médicos de família e comunidade (Quadro 1) e para as pessoas atendidas (Quadro 2), elaborados de forma a contemplar coleta de informaçóes que identificassem componentes do método de abordagem centrada na pessoa. Os questionários foram compostos de perguntas principais e sub-perguntas, sendo que estas visavam a estimular o entrevistado no caso de dificuldades em responder à pergunta principal, não sendo portanto obrigatórias.Assim, cada uma das perguntas principais teve objetivo definido relacionado com os componentes do método de abordagem centrada na pessoa citados anteriormente e com as categorias ou fundamentos teóricos que embasam este estudo. A partir de estudos anteriores ${ }^{2}$ sobre o método clínico centrado na pessoa, definiu-se que seria feita a investigação de três dos seis componentes do método: (1) explorando a doença e a experiência sobre a doença, (2) entendendo a pessoa como um todo, inteira e (3) elaborando um projeto comum ao médico e à pessoa para manejar os problemas. A escolha em trabalhar apenas com estes três componentes deve-se ao fato de que estes seriam os mais representativos do uso de uma abordagem centrada na pessoa e que, nos estudos realizados por Stewart, ${ }^{2}$ revelaram-se mensuráveis.

\section{Resultados e discussão}

O Quadro 3 apresenta as comparaçôes das ideias centrais e dos DSC de médicos e pessoas, que são analisadas a seguir levando-se em consideração as bases teóricas do Método Clínico Centrado na Pessoa.

Ambos os grupos identificam dificuldades de acesso ao cuidado. ${ }^{8} \mathrm{O}$ acesso ao cuidado à saúde é aspecto essencial da APS e da Abordagem Centrada na Pessoa. Historicamente, tem sido um dos dilemas dos serviços de saúde, e muitas propostas têm sido realizadas sobre como organizar este acesso das pessoas de forma que se possa atendê-las de acordo com a sua premência pelo cuidado. ${ }^{9}{ }^{10,11}$ Os DSC de médicos e pessoas confirmam dificuldades relacionadas e caracterizadas por eles como: desproporção entre a oferta de consultas e a demanda, existência das filas, tempo de espera para agendamento e necessidade de persistência para manter continuidade com o mesmo profissional. Mesmo assim, ambos igualmente consideram que o acesso é melhor do que no sistema de saúde, em geral. Um aspecto referido pelos entrevistados e que faz parte de uma Abordagem Centrada na Pessoa, sendo um dos Princípios da APS, é manifestação da existência de vínculo entre pessoa e médico de família, expressando a procura da manutenção da longitudinalidade ${ }^{9}$ do Cuidado por parte de quem busca ajuda ("...] já cria aquele vínculo assim; se não a gente fica desfilando de médico em médico"), embora a autonomia da pessoa de decidir quando e com quem vai ser a consulta esteja de certa forma comprometida.

Os DSC revelam concordância sobre o desconhecimento da Medicina de Família e Comunidade como uma especialidade médica, o que é muito significativo, principalmente numa população que há mais de 20 anos convive com este profissional. Mas embora isto seja importante, mais significativo é o fato de que ambos, médicos de família e pessoas, concordam que o tipo de atendimento prestado nas Unidades do SSC é diferenciado. E as pessoas, na prática, ao descrever o atendimento, identificam características da Medicina de Família e Comunidade, tais como: (1) "Toda a família. Até sogra", (2) "soluciona logo os problemas"; (3) "vêem do lado psicológico até o lado médico mesmo", (4) "a gente se sente à vontade com eles", (5) "e a gente confia", (6) "acho que os pacientes que eu atendo, [...] acho que a grande maioria tem essa percepção da diferença, a gente tenta diferenciar". Um questionamento que merece ser feito é: o desconhecimento da especialidade e da abordagem integral que pode ser realizada pelo médico de família e comunidade por parte de quem busca ajuda não pode fazer com que a pessoa deixe de trazer para a consulta outros aspectos que sejam relevantes, ficando a abordagem centrada em queixas e problemas de um sistema ou aparelho? Neste aspecto, torna-se fundamental uma abordagem centrada na pessoa, que, com 
Quadro 2. Roteiro de entrevistas para pessoas atendidas. ${ }^{6}$

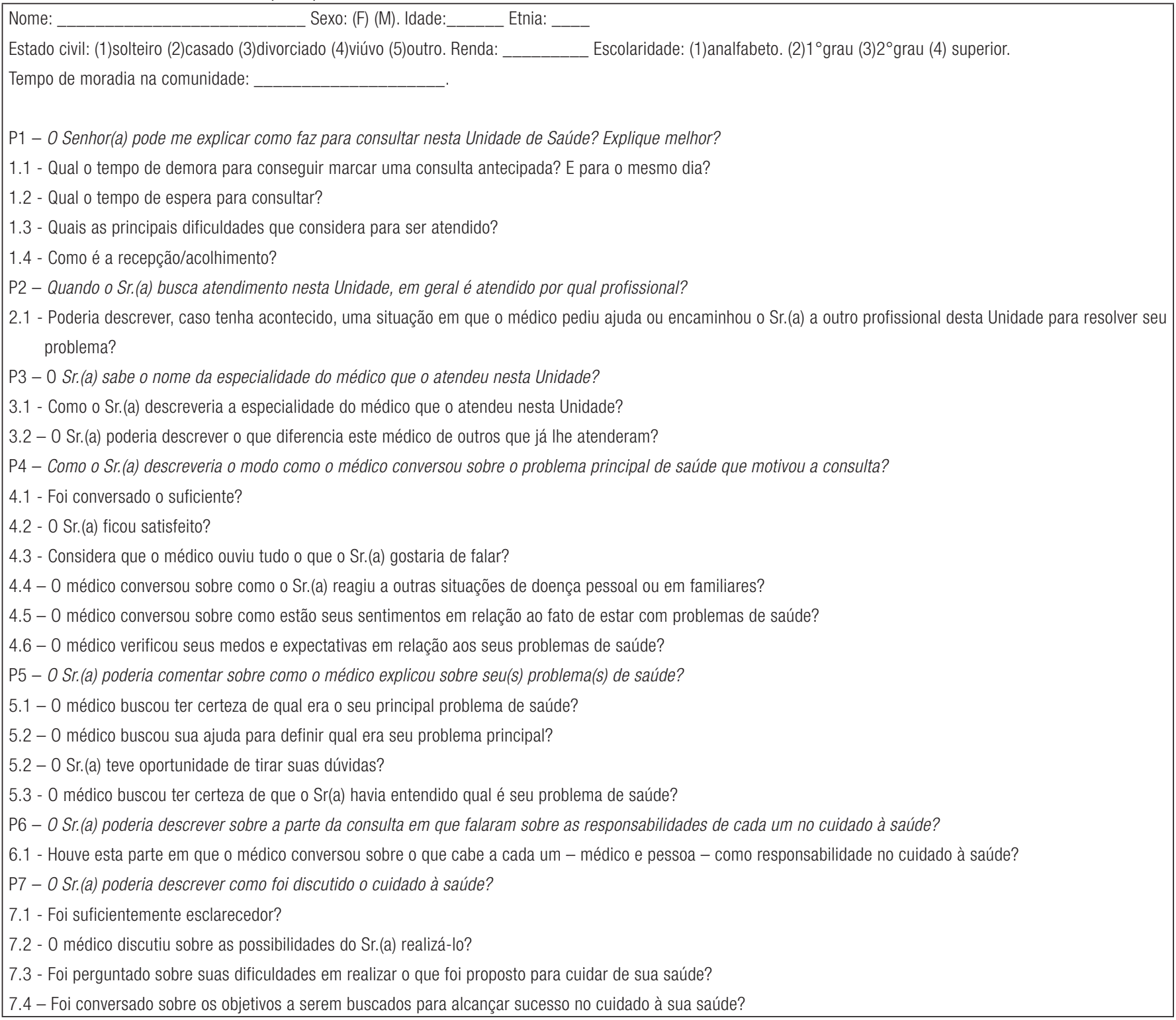

No que se refere ao $1^{\circ}$ componente do MCCP, "Explorando a Doença e a Experiência da pessoa com a Doença”, pode-se afirmar que ele não está de todo presente, ficando a abordagem restrita ao desenvolvimento de uma relação empática e mais focada na queixa verbalizada pela pessoa, correndo-se com isso o risco de abordar a doença (disease) e não a experiência com a doença (illness). ${ }^{14}$ Para se conhecer a pessoa e observar sua autonomia e participaçáo, é importante explorar a narrativa de suas situaçóes prévias de doença.

O $3^{\circ}$ componente, "Buscando um projeto comum ao médico e à pessoa para manejar os problemas", é considerado fundamental para que se caracterize uma abordagem centrada na Pessoa. É o processo através do qual a pessoa e o médico buscam um entendimento e uma concordância mútuos em três aspectos: (1) definindo o problema, (2) estabelecendo objetivos e prioridades de manejo, e (3) identificando os papéis a serem assumidos por ambos. Os resultados dos DSCs mostram que apenas um destes aspectos - a identificação junto à pessoa sobre qual considera seu problema principal - está presente, embora nem sempre explicitado, ao passo que os outros dois aspectos não são claramente introduzidos e trabalhados na consulta. Fica evidenciado que há um entendimento por parte do médico de que as orientaçóes finais da consulta teriam esta função, sendo este então o momento em que a pessoa usaria sua autonomia para manifestar-se em relação ao manejo proposto. Mas, para a pessoa, isto não está claro, ficando muito na dependência do quanto ela se sente à vontade para 


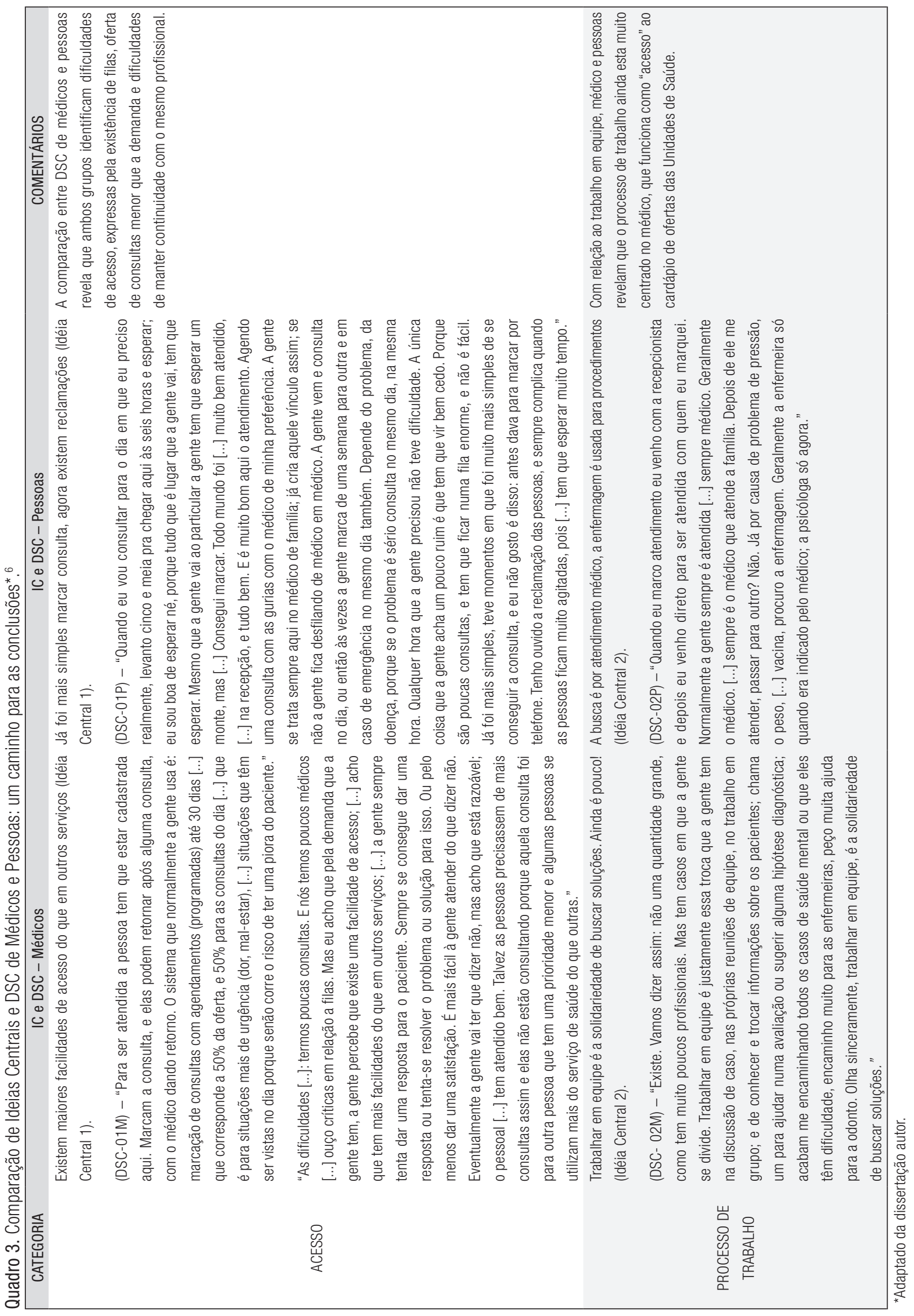




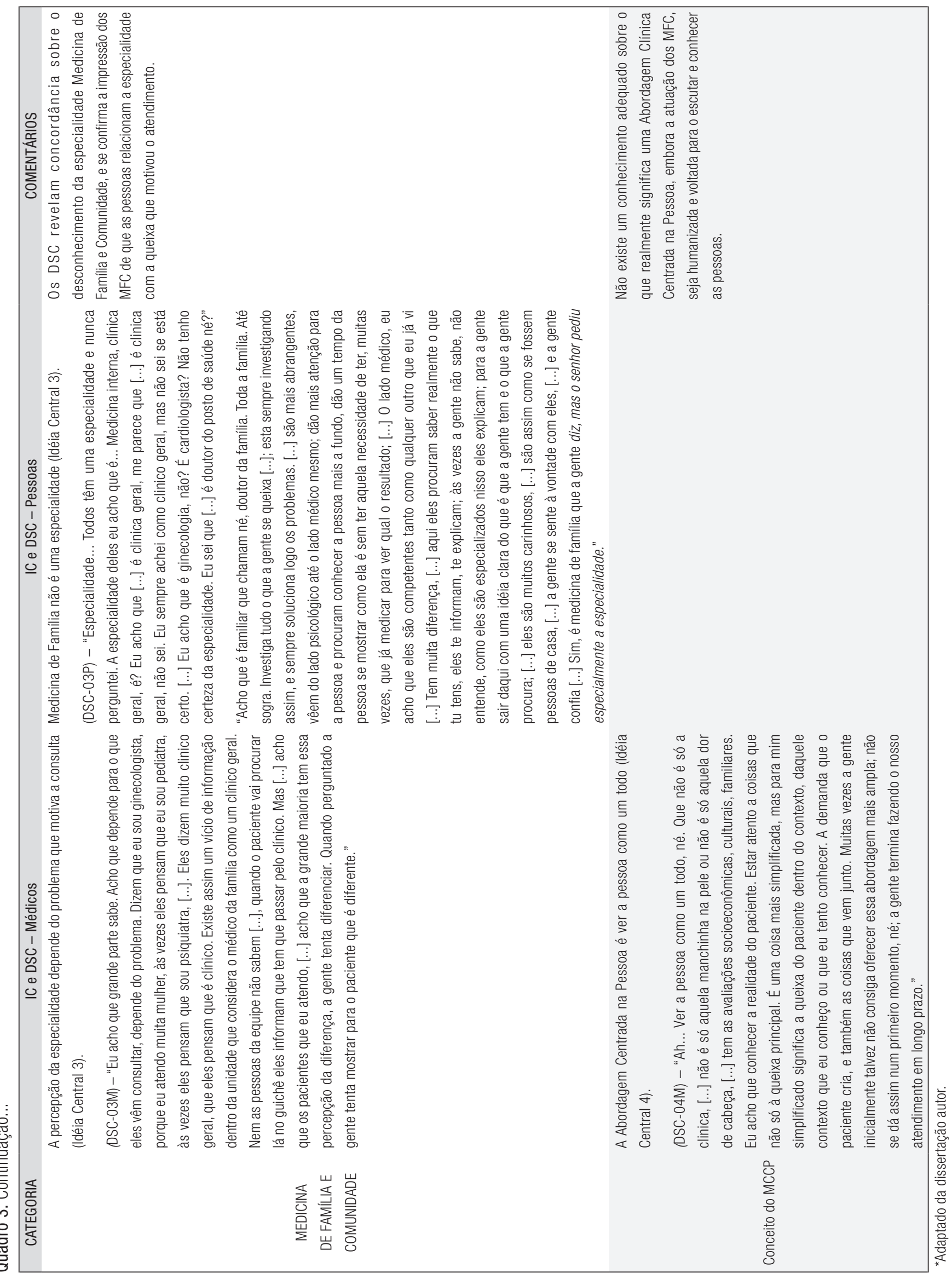




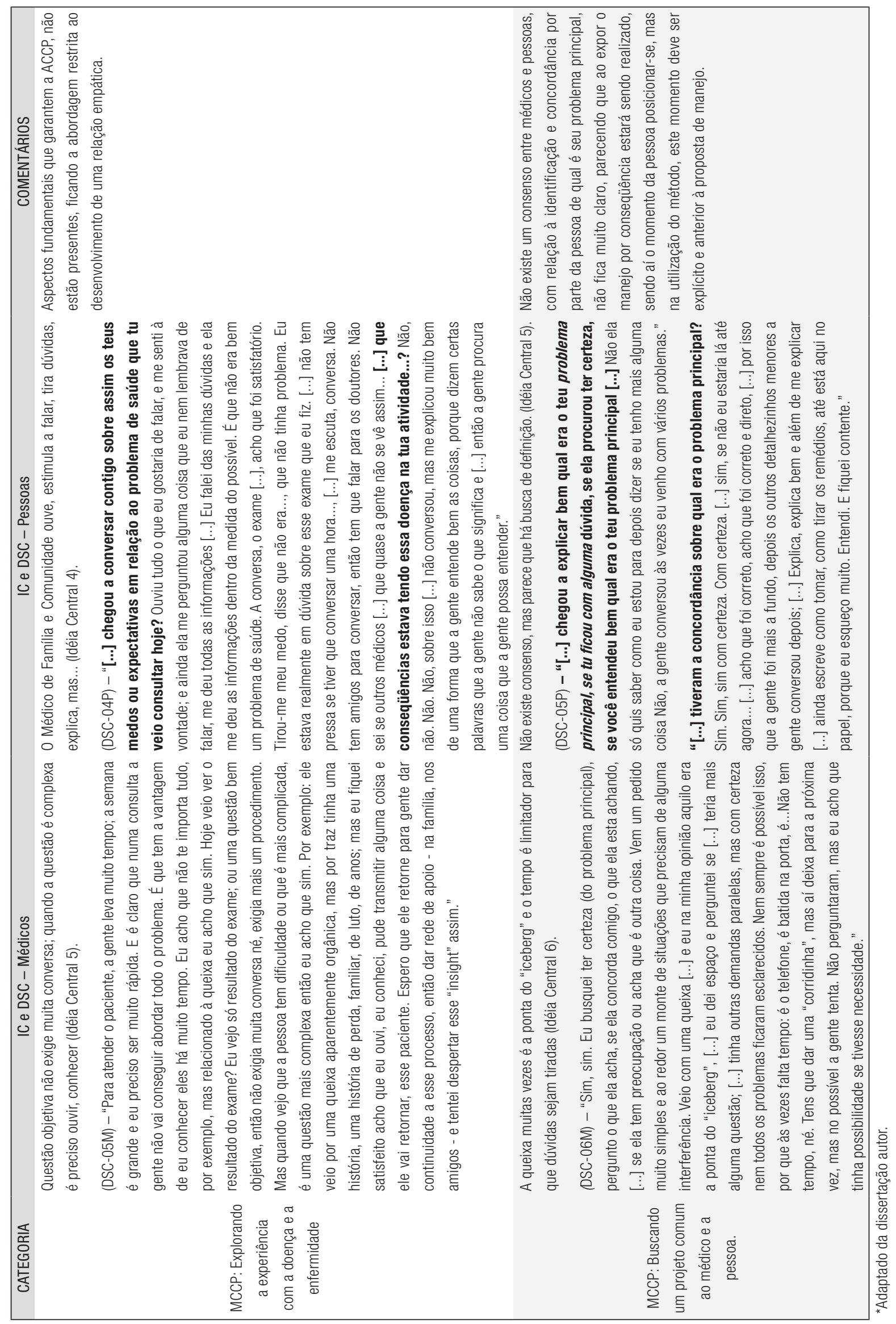




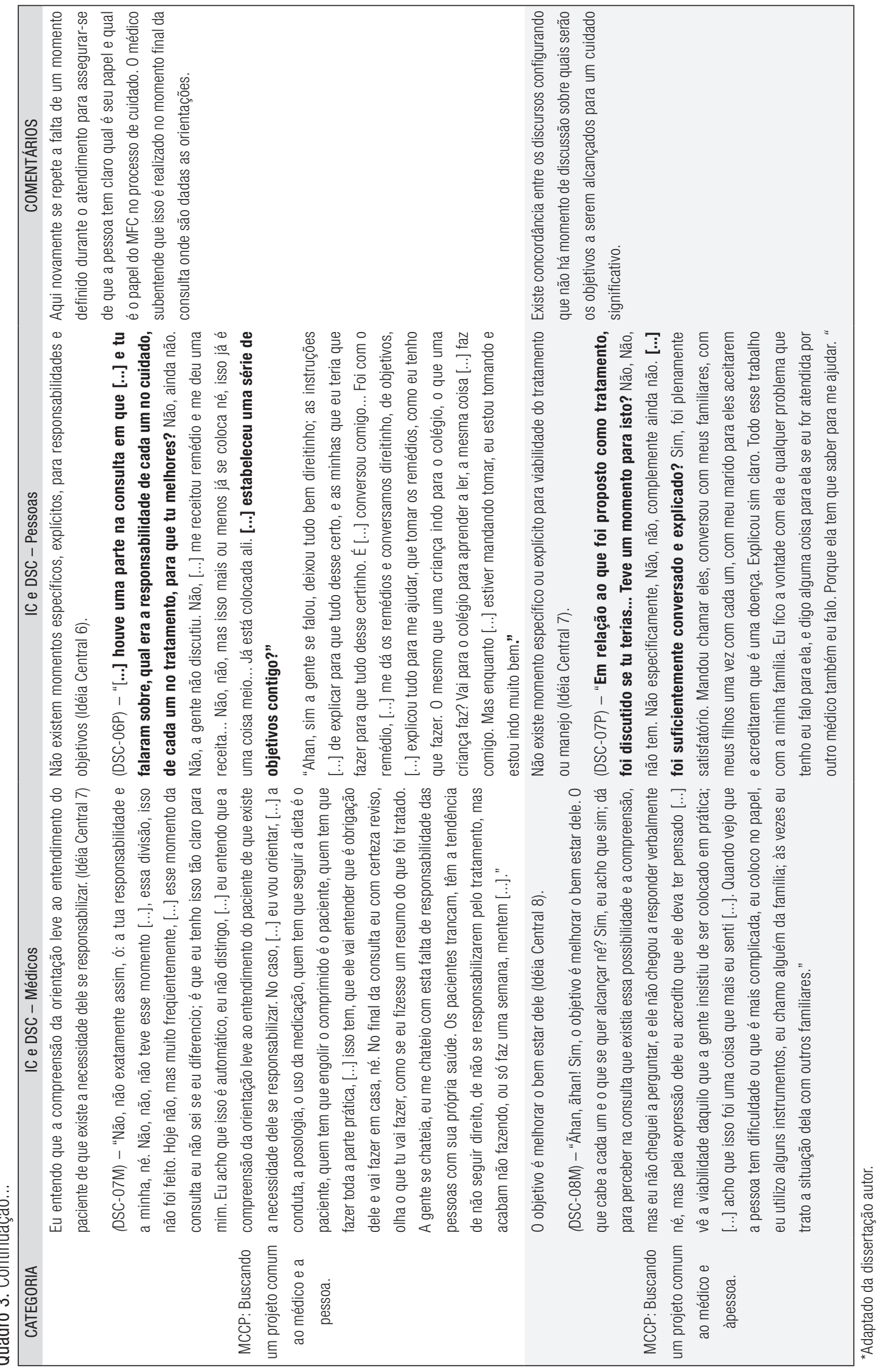




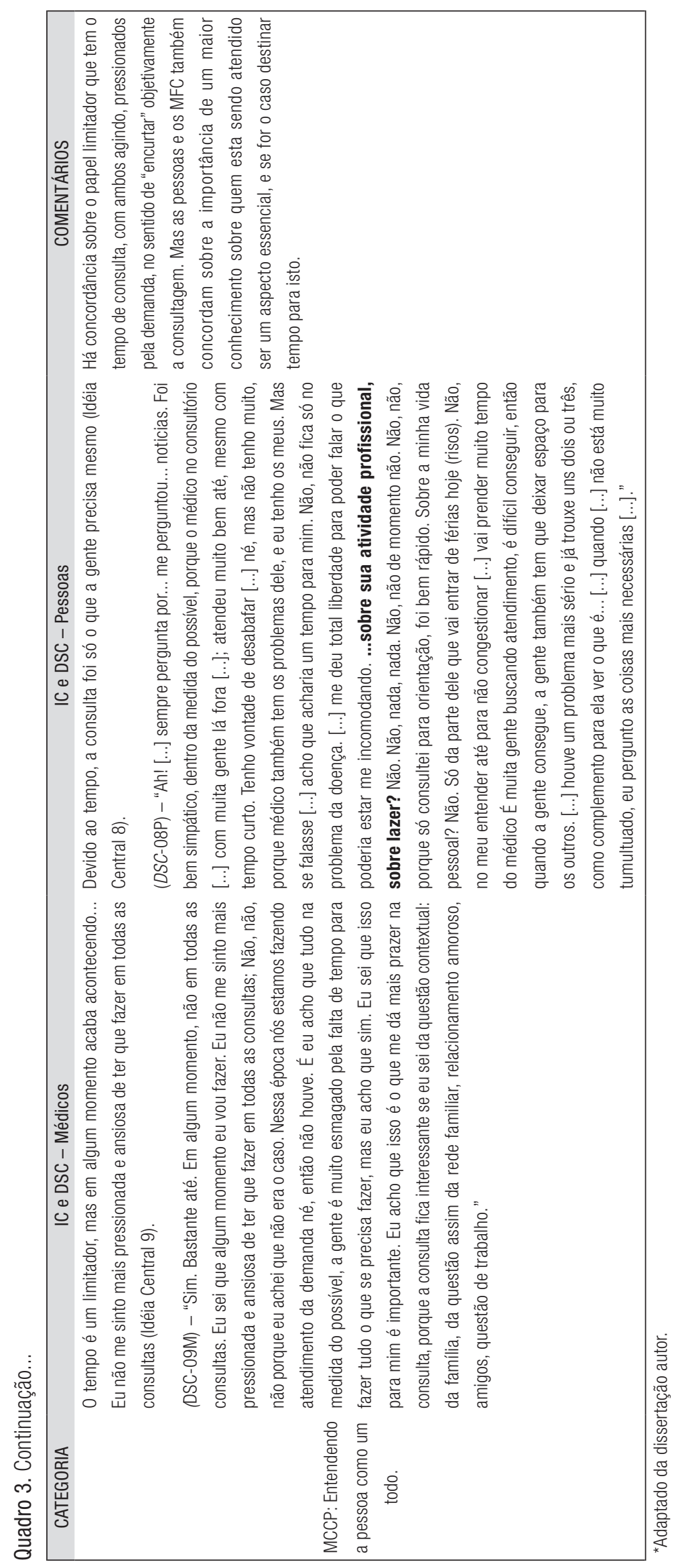


argumentar ou perguntar ao médico. Esta expectativa, de certa forma, configura uma abordagem mais próxima de um modelo informativo ou interpretativo, mas sem conseguir alcançar uma abordagem deliberativa, com uma real participação da pessoa no planejamento de seu cuidado. Aqui repete-se novamente a falta de um momento definido durante o atendimento para assegurar-se de que a pessoa tem claro qual é seu papel e qual é o papel do médico de família e comunidade no processo de cuidado. Existe concordância entre os discursos configurando que não há momento de discussão sobre quais serão os objetivos a serem alcançados para um cuidado significativo. Há concordância sobre o papel limitador que tem o tempo de consulta, com ambos agindo, pressionados pela demanda, no sentido de "encurtar" objetivamente a consulta.

Complementando o que já foi tratado ao discutir a conceituação da Abordagem Centrada na Pessoa pelos médicos de família e comunidade, ao buscar contemplar o $2^{\circ}$ componente do MCCP - "Entendendo a pessoa como um todo, inteira" - devemos ter em conta que as doenças da pessoa são apenas uma dimensão de seus papéis.Portanto elas são um reduzido recurso para entender a doença e o sofrimento da pessoa. Talvez o aspecto mais importante que determina a satisfação com a consulta e que deve ser observado pelo médico não seja a falta de tempo, e sim a indisponibilidade para a pessoa (o que de certa forma é desumano). No caso específico, pode-se perceber que, apesar da falta de tempo, esta indisponibilidade não existe; pelo contrário, as pessoas afirmam que percebem que, se necessário, teriam o tempo do "seu" médico.

Talvez um aspecto inteiramente novo trazido por esta pesquisa tenha sido o de revelar que as pessoas, e não apenas os médicos, são pressionadas pela demanda, pela sala de espera cheia, e utilizam-se do espaço intercessor para exercerem uma parcela de sua autonomia, para limitar-se às questôes mais importantes de sua saúde, numa tentativa solidária de compartilhar o tempo com as demais pessoas que buscam cuidado. Trata-se de uma forma de busca da equidade, por meio do pensamento de que talvez quem está na sala de espera precise de mais tempo do que ela. O entender a pessoa como um todo, inteira, vai muito além do conhecer a pessoa por inteiro, pois envolve, além de obter informações sobre lazer, família, e trabalho, investigar aspectos como os seguintes: (a) desenvolvimento individual (sentido de Eu, auto-estima positiva, independência e autonomia, capacidade de relacionar-se e ter intimidade), (b) fases do desenvolvimento que influenciam a vida das pessoas (posição no ciclo de vida, tarefas que assume, papel que desempenha), (c) Ciclo de Vida pessoal e familiar (pessoas têm um passado, um presente e um futuro!), e (d) o contexto em que estão inseridas (considerar fatores contextuais é uma marca registrada do médico centrado na pessoa). Ao contrário do que se pode pensar, se náo houver uma busca sistemática por estas informaçôes, pensando que o tempo as trará, corre-se o risco de náo realizá-la e acreditar, ao longo do tempo, que já conhecemos o suficiente sobre aquela pessoa. ${ }^{15,16,17}$

\section{Conclusão}

Esta pesquisa permitiu considerar e refletir sobre como é prestado o atendimento por médicos de família e comunidade do SSC-GHC. Ao desenvolver a análise e estabelecer as conclusôes, é importante ter cuidado na hora de extrapolar as observaçôes e limitaçôes existentes. Não obstante, a saturação alcançada permite projetar uma representatividade e significação, possibilitando explorar a relação médico-pessoa em sua plena abrangência. Dessa forma, a partir dos DSC das pessoas e dos médicos, é possível chegar a algumas conclusôes importantes:

a) Em virtude dos relatos, constata-se que existe dificuldade de acesso e, portanto, há uma necessidade de se buscarem maneiras de proporcionar e garantir de forma fácil o acesso das pessoas aos profissionais de saúde, como estratégia primeira para alcançar uma abordagem centrada na pessoa.

b) Podemos constatar que não existe por parte dos médicos de família e comunidade do SSC-GHC utilização de uma abordagem sistematizada, com uma consulta que garanta uma prestação de cuidado centrada na pessoa. Por abordagem sistematizada, entende-se como a forma de desenvolver a consulta ou o atendimento de modo que sempre contemple as habilidades de comunicação necessárias para aquele contexto e situação, de modo a garantir que a abordagem seja centrada na pessoa e alcance os aspectos psicossociais envolvidos.

c) Mesmo assim, percebemos uma satisfação das pessoas atendidas com a consulta realizada pelos médicos de família e comunidade do SSC-GHC, através do reconhecimento explícito da existência de vínculo e da maneira afetiva e carinhosa pela qual se referem aos "seus" médicos. De sua parte, os médicos de família e comunidade também têm a percepção de que prestam um cuidado diferenciado às pessoas, e que buscam se adequar às suas necessidades individuais e familiares ao dispensar este cuidado. Isso com certeza tem a ver com o escutar as pessoas e demonstrar interesse pelos seus problemas, fato que foi ressaltado pelos dois grupos de entrevistados; 
d) Este trabalho também nos permite definir um patamar, um ponto de partida, demonstrando o quanto precisamos avançar e progredir para proporcionar uma formação adequada aos profissionais médicos no uso de tecnologias que levem à Autonomia e ao "entendimento da pessoa como um todo", de fato,realmente conduzindo ao exercício de uma relação "intensificada positivamente" entre médicos e pessoas.

A partir das questóes levantadas por este estudo, podemos apresentar as seguintes sugestóes:

a) Incluir na graduação disciplinas ou campos temáticos sobre os modelos de abordagem médica, que devem ser contemplados com a mesma importância, intensidade, valoração e peso proporcional dos demais conteúdos curriculares tradicionais;

b) Valorizar e aprofundar estes aspectos da atuação do médico de família e comunidade durante a especialização sob forma de Residência Médica,pois, independente da composição multiprofissional das equipes, a consulta é "o momento" principal da prática médica. Também se torna importante para qualquer médico, pois temos verificado que cada vez mais recém-formados em Medicina primeiro realizam uma formação em MFC e depois buscam outras especializaçóes;

c) Proporcionar aos profissionais que já estão atuando no mercado de trabalho uma forma de reciclagem que permita rever sua prática sob uma nova perspectiva de atuação mais voltada para a pessoa;

d) Oportunizar reflexão sobre como permitir à pessoa exercer mais a sua Autonomia no processo de cuidado à saúde. Neste ponto específico, quando se fala em pessoa, está-se referindo tanto ao profissional, que deve poder exercer uma maior participação na organização do seu processo de trabalho e prática, quanto à-pessoa-que-busca-ajuda, que deve poder exercer plenamente seu papel de "especialista nela mesma", contribuindo assim verdadeiramente para obter o melhor e mais adequado em termos de cuidado para seus problemas.

Por fim, a sociedade moderna vive uma crise de conciliação entre o desenvolvimento e a humanização no cuidado à saúde, o que vem abalando a confiança nos médicos.A população está exposta aos danos provocados por remédios ou tratamentos que deveriam curar,à realizaçáo de exames e cirurgias caros e desnecessários, e, muitas vezes, a tratamentos desumanos. A implementação de um método de abordagem que privilegie a pessoa e sua autonomia na busca de um cuidado significativo passa por fazer uma consulta que permita uma visão caleidoscópica de quem está sendo atendido.Na maioria das vezes, o motivo apresentado leva a um diagnóstico óbvio, sendo mais importante conhecer e entender as razóes que levaram a pessoa até o médico e suas relaçóes com a causa ou com o agravamento da doença (illness).Isto somente será alcançado se vermos quem busca ajuda sob diversos ângulos, fazendo um diagnóstico da pessoa. Apesar de parecer simples e óbvio, este é o desafio que tem acompanhado o médico através dos tempos, para que possa realizar intervenção terapêutica multifatorial e interdisciplinar.

\section{Referências}

1. Cassel EJ. The nature of suffering and the goals of medicine. N Engl J Med. 1982;306:639-645. http://dx.doi.org/10.1056/NEJM198203183061104

2. Stewart M. Patiente-centered medicine: transforming the clinical method. $2^{\mathrm{a}}$ ed. Abingdon, United Kingdon: Redcliffe Medical; 2003.

3. Little P. Observational study of effect of patient centredness and positive approach on outcomes of general practice consultations. BMJ. 2001;323(908-911). http://dx.doi.org/10.1136/bmj.323.7318.908

4. Rakel RE. Essentials of family practice. $2^{\text {a }}$ ed. Philadelphia: Saunders; 1998.

5. McWhinney IR. A textebook of family medicine. $2^{\text {a }}$ ed. New York: Oxford University Press; 1997.

6. Lopes JMC. A pessoa como centro do cuidado: a abordagem centrada na pessoa no processo de produção do cuidado médico em Serviço de Atenção Primária à Saúde, 2005 [dissertação]. Rio Grande do Sul: Universidade Federal do Rio Grande do Sul; 2005. Portuguese. Disponível em: http://hdl.handle.net/10183/5873

7. Lefèvre F, Lefèvre AMC. Discurso do sujeito coletivo: um novo enfoque em pesquisa qualitativa (Desdobramentos). Caxias do Sul, RS: EDUCS; 2003.

8. Boff L. Saber cuidar, ética do humano: compaixão pela terra. ga ed. Petrópolis: Vozes; 2003.

9. Starfield B. Atenção primária equilíbrio entre as necessidades de saúde, serviços e tecnologia. Brasília: UNESCO, Ministério da Saúde; 2004.

10. Murray M, Berwick DM. Advanced access: reducing waiting and delays in primary care. Rev. JAMA. 2003;289(8):1035-40.

http://dx.doi.org/10.1001/jama.289.8.1035 
11. Wollmann A, Ros C, Lowen IMV, et al. Novas possibilidades de organizar o acesso e a agenda na Atenção Primária à Saúde. Curitiba: Prefeitura Municipal de Curitiba. Disponível em: http://www.saude.curitiba.pr.gov.br/images/cartilha\%20acesso\%20avan\%C3\%A7ado\%2005_06_14.pdf

12. Chin JJ. Doctor-patient relationship: from medical paternalism to enhanced autonomy. Singapore Med J. 2002;43(3):152-155.

13. Clarke G, Hall RT, Rosencrance G. Physician-patient relations: no more models. Am J Bioeth. 2004;4(2):W16-W19. http://dx.doi.org/10.1162/152651604323097934

14. Coulter A. After Bristol: putting patients at the centre. BMJ. 2002;324:16. http://dx.doi.org/10.1136/bmj.324.7338.648

15. Helman CG. Cultura, saúde e doença. 4ª ed. Porto Alegre: Artmed; 2003.

16. Lown B. A arte perdida de curar. São Paulo: Fundação Petrópolis; 1997.

17. McWhinney IR. Beyond diagnosis: an approach to the integration of behavioural science and clinical medicine. NEJM. 1972;287:384-387. http://dx.doi.org/10.1056/NEJM197208242870805 